\title{
A Composite Finite Element-Finite Difference Model Applied to Turbulence Modelling
}

\author{
Lale Balas and Asu İnan \\ Department of Civil Engineering, Faculty of Engineering and Architecture \\ Gazi University, 06570 Ankara, Turkey \\ lalebal@gazi.edu.tr, \\ asuinan@gazi.edu.tr
}

\begin{abstract}
Turbulence has been modeled by a two equation k- $\omega$ turbulence model to investigate the wind induced circulation patterns in coastal waters. Predictions of the model have been compared by the predictions of two equation $\mathrm{k}-\varepsilon$ turbulence model. Kinetic energy of turbulence is $\mathrm{k}$, dissipation rate of turbulence is $\varepsilon$, and frequency of turbulence is $\omega$. In the three dimensional modeling of turbulence by $\mathrm{k}-\varepsilon$ model and by $\mathrm{k}-\omega$ model, a composite finite element-finite difference method has been used. The governing equations are solved by the Galerkin Weighted Residual Method in the vertical plane and by finite difference approximations in the horizontal plane. The water depths in coastal waters are divided into the same number of layers following the bottom topography. Therefore, the vertical layer thickness is proportional to the local water depth. It has been seen that two equation $\mathrm{k}-\omega$ turbulence model leads to better predictions compared to $\mathrm{k}-\varepsilon$ model in the prediction of wind induced circulation in coastal waters.
\end{abstract}

Keywords: Finite difference, finite element, modeling, turbulence, coastal.

\section{Introduction}

There are different applications of turbulence models in the modeling studies of coastal transport processes. Some of the models use a constant eddy viscosity for the whole flow field, whose value is found from experimental or from trial and error calculations to match the observations to the problem considered. In some of the models, variations in the vertical eddy viscosity are described in algebraic forms. Algebraic or zero equation turbulence models invariably utilize the Boussinesq assumption. In these models mixing length distribution is rather problem dependent and therefore models lack universality. Further problems arise, because the eddy viscosity and diffusivity vanish whenever the mean velocity gradient is zero. To overcome these limitations, turbulence models were developed which accounted for transport or history effects of turbulence quantities by solving differential transport equations for them. In one-equation turbulence models, for velocity scale, the most meaningful scale is $\mathrm{k}^{0.5}$, where $\mathrm{k}$ is the kinetic energy of the turbulent motion per unit mass[1]. In one-equation models, it is difficult to determine the length scale distribution. Therefore the trend has been to move on to two-equation models 
which determine the length scale from a transport equation. One of the two equation models is $\mathrm{k}-\varepsilon$ turbulence model in which the length scale is obtained from the transport equation of dissipation rate of the kinetic energy $\varepsilon$ [2],[3]. The other two equation model is $\mathrm{k}-\omega$ turbulence model that includes two equations for the turbulent kinetic energy $\mathrm{k}$ and for the specific turbulent dissipation rate or the turbulent frequency $\omega$ [4].

\section{Theory}

The implicit baroclinic three dimensional numerical model (HYDROTAM-3), has been improved by a two equation $\mathrm{k}-\omega$ turbulence model. Developed model is capable of computing water levels and water particle velocity distributions in three principal directions by solving the Navier-Stokes equations. The governing hydrodynamic equations in the three dimensional cartesian coordinate system with the z-axis vertically upwards, are [5],[6],[7],[8]:

$$
\begin{gathered}
\frac{\partial \mathrm{u}}{\partial \mathrm{x}}+\frac{\partial \mathrm{v}}{\partial \mathrm{y}}+\frac{\partial \mathrm{w}}{\partial \mathrm{z}}=0 \\
\frac{\partial u}{\partial t}+u \frac{\partial u}{\partial x}+v \frac{\partial u}{\partial y}+w \frac{\partial u}{\partial z}=f v-\frac{1}{\rho_{o}} \frac{\partial p}{\partial x}+2 \frac{\partial}{\partial x}\left(v_{h} \frac{\partial u}{\partial x}\right)+\frac{\partial}{\partial y}\left(v_{h}\left(\frac{\partial u}{\partial y}+\frac{\partial v}{\partial x}\right)\right)+\frac{\partial}{\partial z}\left(v_{z}\left(\frac{\partial u}{\partial z}+\frac{\partial w}{\partial x}\right)\right) \\
\frac{\partial v}{\partial t}+u \frac{\partial v}{\partial x}+v \frac{\partial v}{\partial y}+w \frac{\partial v}{\partial z}=-f u-\frac{1}{\rho_{o} \partial y}+2 \frac{\partial}{\partial y}\left(v_{h} \frac{\partial v}{\partial y}\right)+\frac{\partial}{\partial x}\left(v_{h}\left(\frac{\partial v}{\partial x}+\frac{\partial u}{\partial y}\right)\right)+\frac{\partial}{\partial z}\left(v_{z}\left(\frac{\partial v}{\partial z}+\frac{\partial w}{\partial y}\right)\right) \\
\frac{\partial w}{\partial t}+u \frac{\partial w}{\partial x}+v \frac{\partial w}{\partial y}+w \frac{\partial w}{\partial z}=-\frac{1}{\rho_{o} \partial z}-g+\frac{\partial}{\partial y}\left(v_{h}\left(\frac{\partial w}{\partial y}+\frac{\partial v}{\partial z}\right)\right)+\frac{\partial}{\partial x}\left(v_{h}\left(\frac{\partial w}{\partial x}+\frac{\partial u}{\partial z}\right)\right)+\frac{\partial}{\partial z}\left(v_{z} \frac{\partial w}{\partial z}\right)
\end{gathered}
$$

where, $\mathrm{x}, \mathrm{y}$ :horizontal coordinates, z:vertical coordinate, t:time, u,v,w:velocity components in $\mathrm{x}, \mathrm{y}, \mathrm{z}$ directions at any grid locations in space, $\mathrm{v}_{\mathrm{z}}$ :eddy viscosity coefficients in $\mathrm{z}$ direction, $\mathrm{v}_{\mathrm{h}}$ :horizontal eddy viscosity coefficient, f:corriolis coefficient, $\rho(\mathrm{x}, \mathrm{y}, \mathrm{z}, \mathrm{t})$ :water density, g:gravitational acceleration, $\mathrm{p}$ :pressure.

As the turbulence model, firstly, modified k- $\omega$ turbulence model is used. Model includes two equations for the turbulent kinetic energy $\mathrm{k}$ and for the specific turbulent dissipation rate or the turbulent frequency $\omega$. Equations of $k-\omega$ turbulence model are given by the followings.

$$
\begin{gathered}
\frac{d k}{d t}=\frac{\partial}{\partial z}\left[\sigma^{*} v_{z} \frac{\partial k}{\partial z}\right]+P+\frac{\partial}{\partial x}\left[\sigma^{*} v_{h} \frac{\partial k}{\partial x}\right]+\frac{\partial}{\partial y}\left[\sigma^{*} v_{h} \frac{\partial k}{\partial y}\right]-\beta^{*} \varpi k \\
\frac{d \varpi}{d t}=\frac{\partial}{\partial z}\left[\sigma^{*} v_{z} \frac{\partial \varpi}{\partial z}\right]+\alpha \frac{\varpi}{k} P+\frac{\partial}{\partial x}\left[\sigma^{*} v_{h} \frac{\partial \varpi}{\partial x}\right]+\frac{\partial}{\partial y}\left[\sigma^{*} v_{h} \frac{\partial \varpi}{\partial y}\right]-\beta \varpi^{2}
\end{gathered}
$$


The stress production of the kinetic energy $\mathrm{P}$, and eddy viscosity $\mathrm{v}_{\mathrm{z}}$ are defined by;

$$
P=v_{h}\left[2\left(\frac{\partial u}{\partial x}\right)^{2}+2\left(\frac{\partial v}{\partial y}\right)^{2}+\left(\frac{\partial u}{\partial y}+\frac{\partial v}{\partial x}\right)^{2}\right]+v_{z}\left[\left(\frac{\partial u}{\partial z}\right)^{2}+\left(\frac{\partial v}{\partial z}\right)^{2}\right] ; \quad v_{z}=\frac{k}{\varpi}
$$

At high Reynolds Numbers $\left(\mathrm{R}_{\mathrm{T}}\right)$, the constants are used as; $\alpha=5 / 9, \beta=3 / 40, \beta^{*}=$ $9 / 100, \sigma=1 / 2$ and $\sigma^{*}=1 / 2$. Whereas at lower Reynolds numbers they are calculated as;

$$
\alpha^{*}=\frac{1 / 40+R_{T} / 6}{1+R_{T} / 6} ; \alpha=\frac{5}{9} \frac{1 / 10+R_{T} / 2.7}{1+R_{T} / 2.7}\left(\alpha^{*}\right)^{-1} ; R_{T}=\frac{k}{\varpi v} ; \beta^{*}=\frac{9}{100} \frac{5 / 18+\left(R_{T} / 8\right)^{4}}{1+\left(R_{T} / 8\right)^{4}}
$$

Secondly, as the turbulence model a two equation $k-\varepsilon$ model has been applied. Equations of $\mathrm{k}-\varepsilon$ turbulence model are given by the followings.

$$
\begin{gathered}
\frac{\partial k}{\partial t}+u \frac{\partial k}{\partial x}+v \frac{\partial k}{\partial y}+w \frac{\partial k}{\partial z}=\frac{\partial}{\partial z}\left(\frac{v_{z}}{\sigma_{k}} \frac{\partial k}{\partial z}\right)+P-\varepsilon+\frac{\partial}{\partial x}\left(v_{h} \frac{\partial k}{\partial x}\right)+\frac{\partial}{\partial y}\left(v_{h} \frac{\partial k}{\partial y}\right) \\
\frac{\partial \varepsilon}{\partial t}+u \frac{\partial \varepsilon}{\partial x}+v \frac{\partial \varepsilon}{\partial y}+w \frac{\partial \varepsilon}{\partial z}=\frac{\partial}{\partial z}\left(\frac{v_{z}}{\sigma_{\varepsilon}} \frac{\partial \varepsilon}{\partial z}\right)+C_{1 \varepsilon} P \frac{\varepsilon}{k}-C_{2 \varepsilon} \frac{\varepsilon^{2}}{k}+\frac{\partial}{\partial x}\left(v_{h} \frac{\partial \varepsilon}{\partial x}\right)+\frac{\partial}{\partial y}\left(v_{h} \frac{\partial \varepsilon}{\partial y}\right)
\end{gathered}
$$

where, $\mathrm{k}$ :Kinetic energy, $\varepsilon:$ Rate of dissipation of kinetic energy, P: Stress production of the kinetic energy. The following universal $\mathrm{k}-\varepsilon$ turbulence model empirical constants are used and the vertical eddy viscosity is calculated by:

$$
v_{z}=C_{\mu} \frac{k^{2}}{\varepsilon} ; \mathrm{C}_{\mu}=0.09, \sigma_{\varepsilon}=1.3, \mathrm{C}_{1 \varepsilon}=1.44, \mathrm{C}_{2 \varepsilon}=1.92 .
$$

Some other turbulence models have also been widely applied in three dimensional numerical modeling of wind induced currents such as one equation turbulence model and mixing length models. They are also used in the developed model HYROTAM3 , however it is seen that two equation turbulence models give better predictions compared to the others.

\section{Solution Method}

Solution method is a composite finite difference-finite element method. Equations are solved numerically by approximating the horizontal gradient terms using a staggered finite difference scheme (Fig.1a). In the vertical plane however, the Galerkin Method of finite elements is utilized. Water depths are divided into the same number of layers following the bottom topography (Fig.1b). At all nodal points, the ratio of the length (thickness) of each element (layer) to the total depth is constant. The mesh size may be varied in the horizontal plane. By following the finite element approach, all the variables at any point over the depth are written in terms of the discrete values of these variables at the vertical nodal points by using linear shape functions. 


$$
\tilde{G}=N_{1} G_{1}^{k}+N_{2} G_{2}^{k} ; N_{1}=\frac{z_{2}-z}{l_{k}} ; N_{2}=\frac{z-z_{1}}{l_{k}} ; l_{k}=z_{2}-z_{1}
$$

where $\tilde{G}$ :shape function; G: any of the variables, $k$ : element number; $N_{1}, N_{2}$ linear interpolation functions; $l_{k}$ :length of the $k$ 'th element; $z_{1}, z_{2}$ :beginning and end elevations of the element $\mathrm{k}$; $\mathrm{z}$ : transformed variable that changes from $z_{1}$ to $z_{2}$ in an element.

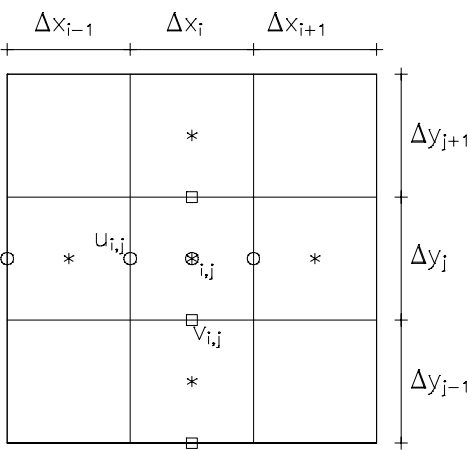

(a)

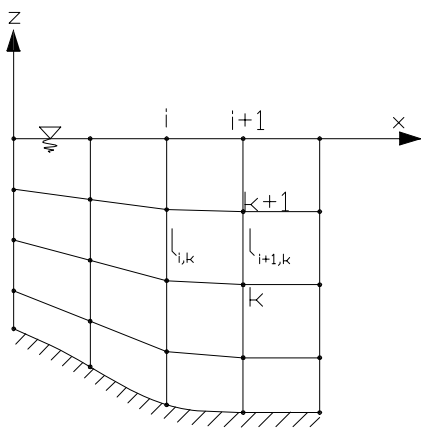

(b)

Fig. 1. a) Horizontal staggered finite difference scheme, o: longitudinal horizontal velocity, u; 口: lateral horizontal velocity, v; *: all other variables b) Finite element scheme throughout the water depth

After the application of the Galerkin Method, any derivative terms with respect to horizontal coordinates appearing in the equations are replaced by their central finite difference approximations. The system of nonlinear equations is solved by the Crank Nicholson Method which has second order accuracy in time. Some of the finite difference approximations are given in the following equations.

$$
\begin{aligned}
& \left(\frac{\partial l}{\partial x}\right)_{i, j}=\frac{\left(\Delta x_{i}+\Delta x_{i+1}\right)\left(l_{i, j}-l_{i-1, j}\right)}{\left(\Delta x_{i}+\Delta x_{i-1}\right)\left(\Delta x_{i}+\frac{\Delta x_{i+1}+\Delta x_{i-1}}{2}\right)}+\frac{\left(\Delta x_{i}+\Delta x_{i-1}\right)\left(l_{i+1, j}-l_{i, j}\right)}{\left(\Delta x_{i}+\Delta x_{i+1}\right)\left(\Delta x_{i}+\frac{\Delta x_{i+1}+\Delta x_{i-1}}{2}\right)} \\
& \left(\frac{\partial l}{\partial y}\right)_{i, j}=\frac{\left(\Delta y_{j}+\Delta y_{j+1}\right)\left(l_{i, j}-l_{i, j-1}\right)}{\left(\Delta y_{j}+\Delta y_{j-1}\right)\left(\Delta y_{j}+\frac{\Delta y_{j-1}+\Delta y_{j+1}}{2}\right)}+\frac{\left(\Delta y_{j}+\Delta y_{j-1}\right)\left(l_{i, j+1}-l_{i, j}\right)}{\left(\Delta y_{j}+\Delta y_{j+1}\right)\left(\Delta y_{j}+\frac{\Delta y_{j-1}+\Delta y_{j+1}}{2}\right)} \\
& \left(\frac{\partial C}{\partial x}\right)_{i, j}=\frac{\left(\Delta x_{i}+\Delta x_{i+1}\right)\left(C_{i, j}-C_{i-1, j}\right)}{\left(\Delta x_{i}+\Delta x_{i-1}\right)\left(\Delta x_{i}+\frac{\Delta x_{i+1}+\Delta x_{i-1}}{2}\right)}+\frac{\left(\Delta x_{i}+\Delta x_{i-1}\right)\left(C_{i+1, j}-C_{i, j}\right)}{\left(\Delta x_{i}+\Delta x_{i+1}\right)\left(\Delta x_{i}+\frac{\Delta x_{i+1}+\Delta x_{i-1}}{2}\right)}
\end{aligned}
$$




$$
\begin{gathered}
\left(\frac{\partial C}{\partial y}\right)_{i, j}=\frac{\left(\Delta y_{j}+\Delta y_{j+1}\right)\left(C_{i, j}-C_{i, j-1}\right)}{\left(\Delta y_{j}+\Delta y_{j-1}\right)\left(\Delta y_{j}+\frac{\Delta y_{j-1}+\Delta y_{j+1}}{2}\right)}+\frac{\left(\Delta y_{j}+\Delta y_{j-1}\right)\left(C_{i, j+1}-C_{i, j}\right)}{\left(\Delta y_{j}+\Delta y_{j+1}\right)\left(\Delta y_{j}+\frac{\Delta y_{j-1}+\Delta y_{j+1}}{2}\right)} \\
\left(\frac{\partial^{2} C}{\partial y^{2}}\right)_{i, j}=2\left(\frac{C_{i, j-1}}{\frac{\left(\Delta y_{j}+\Delta y_{j-1}\right)\left(\Delta y_{j}+\frac{\Delta y_{j-1}+\Delta y_{j+1}}{2}\right)}{2} \frac{\left(\Delta y_{j}+\Delta y_{j-1}\right)\left(\Delta y_{j}+\Delta y_{j+1}\right)}{2}}\right) \\
+\frac{C_{i, j+1}}{2}\left(\Delta y_{j}+\Delta y_{j+1}\right)\left(\frac{\left.\Delta y_{j+1}+\Delta y_{j-1}\right)}{2}\right)
\end{gathered}
$$

\section{Model Applications}

Simulated velocity profiles by using k- $\varepsilon$ turbulence model, $\mathrm{k}-\omega$ turbulence model have been compared with the experimental results of wind driven turbulent flow of an homogeneous fluid conducted by Tsanis and Leutheusser [9]. Laboratory basin had a length of $2.4 \mathrm{~m}$., a width of $0.72 \mathrm{~m}$. and depth of $\mathrm{H}=0.05$ meters. The Reynolds Number, $R_{s}=\frac{u_{s} H \rho}{\mu}$ was 3000 ( $\mathrm{u}_{\mathrm{s}}$ is the surface velocity, $\mathrm{H}$ is the depth of the flow, $\rho$ is the density of water and $\mu$ is the dynamic viscosity). The velocity profiles obtained by using k- $\varepsilon$ turbulence model and k- $\omega$ turbulence model are compared with the measurements in Fig.2a and vertical eddy viscosity distributions are given in Fig.2b. 


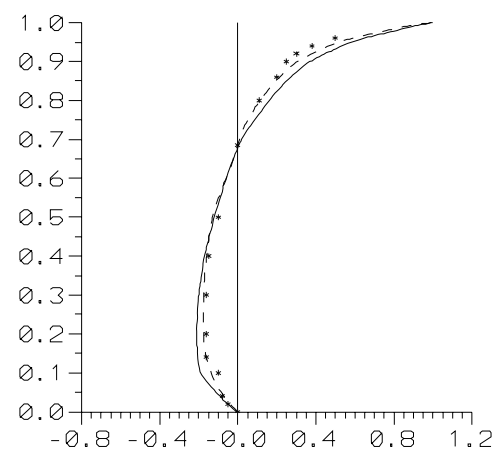

(a)

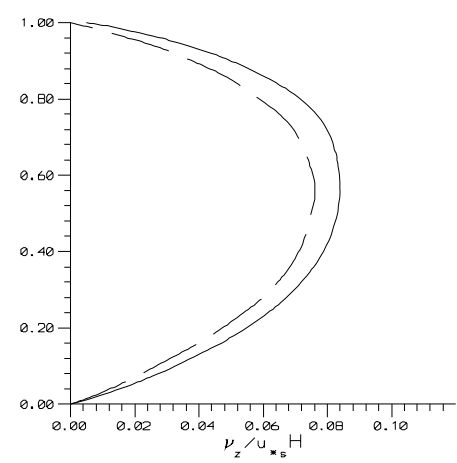

(b)

Fig. 2. a)Velocity profiles, b) Distribution of vertical eddy viscosity (solid line: k- $\varepsilon$ turbulence model, dashed line: k- $\omega$ turbulence model, *: experimental data)

The root mean square error of the nondimensional horizontal velocity predicted by the $\mathrm{k}-\varepsilon$ turbulence model is 0.08 , whereas it drops to 0.02 in the predictions by using $\mathrm{k}-\omega$ turbulence model. This basically due to a better estimation of vertical distribution of vertical eddy viscosity by $\mathrm{k}-\omega$ turbulence model.

Developed three dimensional numerical model (HYROTAM-3) has been implemented to Bay of Fethiye located at the Mediterranean coast of Turkey. Water depths in the Bay are plotted in Fig.3a. The grid system used has a square mesh size of $100 \times 100 \mathrm{~m}$. Wind characteristics are obtained from the measurements of the meteorological station in Fethiye for the period of 1980-2002. The wind analysis shows that the critical wind direction for wind speeds more than $7 \mathrm{~m} / \mathrm{s}$, is WNWWSW direction. Some field measurements have been performed in the area. The current pattern over the area is observed by tracking drogues, which are moved by currents at the water depths of $1 \mathrm{~m} ., 5 \mathrm{~m}$ and $10 \mathrm{~m}$.. At Station I and at Station II shown in Fig.3a, continuous velocity measurements throughout water depth, at Station III water level measurements were taken for 27 days. In the application measurement period has been simulated and model is forced by the recorded wind as shown in Fig. 3b. No significant density stratification was recorded at the site. Therefore water density is taken as a constant. A horizontal grid spacing of $\Delta \mathrm{x}=\Delta \mathrm{y}=100 \mathrm{~m}$. is used. Horizontal eddy viscosities are calculated by the sub-grid scale turbulence model and the vertical eddy viscosity is calculated by k- $\varepsilon$ turbulence model and also by k- $\omega$ turbulence model. The sea bottom is treated as a rigid boundary. Model predictions are in good agreement with the measurements. Simulated velocity profiles over the depth at the end of 4 days are compared with the measurements taken at Station I and Station II and are shown in Fig.4. At Station I, the root mean square error of the horizontal velocity is $0.19 \mathrm{~cm} / \mathrm{s}$ in the predictions by $\mathrm{k}$ $\varepsilon$ turbulence model and it is $0.11 \mathrm{~cm} / \mathrm{s}$ in the predictions by $\mathrm{k}-\omega$ turbulence model. At Station II, the root mean square error of the horizontal velocity is $0.16 \mathrm{~cm} / \mathrm{s}$ in the predictions by $\mathrm{k}-\varepsilon$ turbulence model and it is $0.09 \mathrm{~cm} / \mathrm{s}$ in the predictions by $\mathrm{k}-\omega$ turbulence model. 

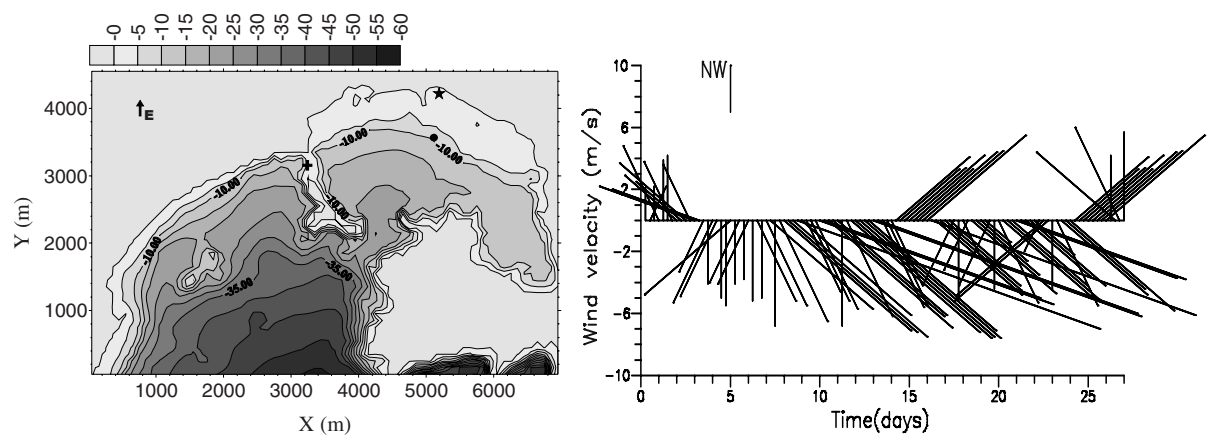

Fig. 3. a)Water depths (m) of Fethiye Bay, + :Station I, $\bullet:$ Station II, $*$ :Station III. b) Wind speeds and directions during the measurement period.

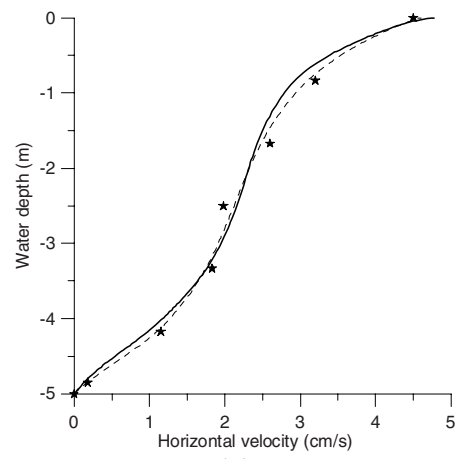

(a)

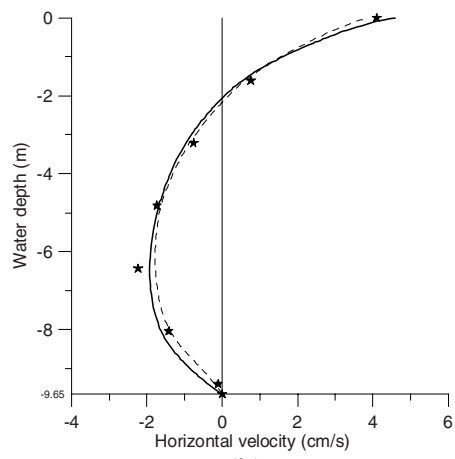

(b)

Fig. 4. Simulated velocity profiles over the depth at the end of 4 days; solid line: k- $\varepsilon$ turbulence model, dashed line: k- $\omega$ turbulence model, *: experimental data, a) at Station I, b) at Station II

\section{Conclusions}

From the two equation turbulence models, k- $\varepsilon$ model and k- $\omega$ model have been used in three dimensional modeling of coastal flows. The main source of coastal turbulence production is the surface current shear stress generated by the wind action. In the numerical solution a composite finite element-finite difference method has been applied. Governing equations are solved by the Galerkin Weighted Residual Method in the vertical plane and by finite difference approximations in the horizontal plane on a staggered scheme. Generally, two-equation turbulence models give improved estimations compared to other turbulence models. In the comparisons of model predictions with both the experimental and field measurements, it is seen that the two equation $\mathrm{k}-\omega$ turbulence model predictions are better than the predictions of two equation $\mathrm{k}-\varepsilon$ turbulence model. This is basically due to the better parameterizations of the nonlinear processes in the formulations leading a more reliable and numerically rather easy to handle vertical eddy viscosity distribution in the k- $\omega$ turbulence model. 
Acknowledgment. The author wishes to thank the anonymous referees for their careful reading of the manuscript and their fruitful comments and suggestions.

\section{References}

1. Li, Z., Davies, A.G.: Turbulence Closure Modelling of Sediment Transport Beneath Large Waves. Continental Shelf Research (2001) 243-262

2. Bonnet-Verdier,C., Angot P., Fraunie, P., Coantic, M.: Three Dimensional Modelling of Coastal Circulations with Different k- $\varepsilon$ Closures. Journal of Marine Systems (2006) 321339

3. Baumert, H., Peters, H.: Turbulence Closure, Steady State, and Collapse into Waves. Journal of Physical Oceanography 34 (2004) 505-512

4. Neary, V.S., Sotiropoulos, F., Odgaard, A.J.: Three Dimensional Numerical Model of Lateral Intake Inflows. Journal of Hyraulic Engineering 125 (1999) 126-140

5. Balas,L., Özhan, E.: An Implicit Three Dimensional Numerical Model to Simulate Transport Processes in Coastal Water Bodies, International Journal for Numerical Methods in Fluids 34 (2000) 307-339

6. Balas, L., Özhan, E.: Three Dimensional Modelling of Stratified Coastal Waters, Estuarine, Coastal and Shelf Science 56 (2002) 75-87

7. Balas, L.: Simulation of Pollutant Transport in Marmaris Bay. China Ocean Engineering, Nanjing Hydraulics Research Institute (NHRI) 15 (2001) 565-578

8. Balas, L., Özhan, E: A Baroclinic Three Dimensional Numerical Model Applied to Coastal Lagoons. Lecture Notes in Computer Science 2658 (2003) 205-212

9. Tsanis,K.I., Leutheusser, H.J.:The Structure of Turbulent Shear-Induced Countercurrent Flow, Journal of Fluid Mechanics 189 (1998) 531-552 